\title{
Dynamic 3D Clustering of Spatio-Temporal Brain Data in the NeuCube Spiking Neural Network Architecture on a Case Study of fMRI Data
}

\author{
Maryam Gholami-Doborjeh, Nikola Kasabov \\ Knowledge Engineering and Discovery Research Institute \\ Auckland University of Technology \\ Auckland, New Zealand \\ mgholamieaut.ac.nz, nkasaboveaut.ac.nz
}

\begin{abstract}
The paper presents a novel clustering method for dynamic SpatioTemporal Brain Data (STBD) on the case study of functional Magnetic Resonance Image (fMRI). The method is based on NeuCube spiking neural network (SNN) architecture, where the spatio-temporal relationships between STBD streams are learned and simultaneously the clusters are created. The clusters are represented as groups of spiking neurons inside the NeuCube's spiking neural network cube (SNNc). The centroids of the clusters are predefined by spatial location of the brain data sources used as input variables. We illustrate the proposed clustering method on an fMRI case study STBD recorded during a cognitive task. A comparative analysis of the clusters across different mental activities can reveal new findings about the brain processes under study.
\end{abstract}

\section{Introduction}

The human brain is acting as a complex information processing machine [1], [2], that processes information throughout communications between billions of neurons. In order to analyse STBD, suitable models are needed to trace such complex patterns and to understand mental processes that generate the data. In this paper we use the NeuCube framework [3] as a rich model for modelling and understanding of STBD. We propose a new method for dynamic clustering of STBD in a NeuCube model and illustrate it on fMRI data.

\section{The Proposed NeuCube-Based Spiking Neural Network Methodology for Learning, Visualisation and Clustering of STBD}

\subsection{Spiking Neural Networks for Modelling STBD}

Spiking neural networks model operation is based on neuron synaptic states that incorporate spiking time. SNNs have become popular computational methods for 
complex spatio/spectro temporal data analysis [3]. Their neuromorphic highly parallel hardware implementations are advancing very fast [4].

\subsection{The NeuCube Architecture [3]}

NeuCube is an evolving spiking neural network (eSNN) architecture for STBD learning, modelling, knowledge extraction, and for the analysis of the brain processes that generated the data [3]. The NeuCube architecture consists of several modules:

An Input encoding module for transforming spatio-temporal data into spike trains; A 3D SNN cube (SNNc) module for unsupervised learning of STBD; An output classification/regression module for supervised learning of data; Optimisation module; Visualisation and knowledge extraction module. Based on the NeuCube architecture we propose here a new clustering method schematically represented in Fig. 1.

\subsection{D Dynamic Neuronal Clustering in a NeuCube SNN Model}

The proposed STBD clustering method includes the following steps:

Step 1: STBD recording, i.e. for a given problem, relevant STBD is recorded.

Step 2: STBD encoding and mapping into a 3D SNNc as part of a NeuCube model.

Step 3: Unsupervised learning in the 3D SNNc and cluster evolution based on the spiking activity and connectivity in SNNc.

Step 4: Analysis of the connectivity of the trained 3D SNNc as dynamic spatiotemporal clusters of the STBD.

Step 5: Mapping SNNc clusters into brain regions.

Step 6: Functional analysis of the dynamic interaction between SNNc clusters to understand brain functional dynamics.

At Step 2, STBD is encoded into spike trains using an appropriate encoding algorithm such as Threshold-Based Representation method (TBR). The spike trains are then entered into a 3D SNNc which is created with a suitable size to map the brain template relevant to the data, such as Talairach [6], MNI [7], or voxel coordinates of individual brain data. Input neurons are allocated in the SNNc to enter input variable spike trains. The SNNc is initialised using a small world connectivity rule [7].

At Step 3, spike trains are learned in a SNNc using Spike-Timing Dependent Plasticity (STDP) learning rule [8]. During the learning procedure, spatio-temporal relationships within data are captured in the form of evolving connection weights and simultaneously neurons are clustered into homogeneous groups with respect to the neurons' activation similarity. Formation of the clusters is based on the intensity of spike communication within the SNNc as a similarity measure. The similarity measure is not only based on the spatial information of the data, but also on the temporal similarity as spike-time relationships. The results are dynamic 3D clusters containing the most evoked neurons by the corresponding cluster's centroid. The clusters represent spiking neurons with similar spiking activity at each time, reflecting dynamic spatiotemporal brain processes. The proposed clustering method differs significantly from the existing methods for clustering, such as evolving clustering (e.g. DENFIS [9] which 
method deals with static data and no temporal relationships between input vectors are learned) or self-organising maps [10].

\section{Application of the Proposed Method on a Benchmark fMRI STBD}

To demonstrate our clustering method, the known STAR/PLUS fMRI data [11] is used to study how neurons in the SNNc are clustered based on the spatio-temporal similarity measure of the neurons' activation against different sentence polarities.

\section{1 fMRI Data Acquisition Description}

In STAR/PLUS fMRI data, the whole brain volume is recorded every 500 milliseconds, while a cognitive task (pictures/sentences stimuli matching) performed by a subject. When the subject reads a sentence, the brain activity patterns are performed differently depending on the sentence polarity (affirmative $v s$. negative). In this experiment, fMRI STBD is divided into two time series corresponding to the two classes: Class 1, reading affirmative sentences; Class 2, reading negative sentences. In this study, the most activated voxels against sentence stimuli are selected using Signal-to-Noise Ratio (SNR) feature selection as reported in Table I.

Table 1. The more activated ROIs are presented in decreasing order of SNR values. In bracket is the number of voxels located in every region

\begin{tabular}{|l|c|c|c|c|c|}
\hline \multicolumn{6}{|c|}{ Activated brain region (number of selected voxels) } \\
\hline $\mathbf{1}$ & 'LT' (3) & $\mathbf{4}$ & 'LDLPFC' (6) & $\mathbf{7}$ & 'RDLPFC'(1) \\
\hline $\mathbf{2}$ & 'LOPER' (3) & $\mathbf{5}$ & 'RT'(2) & $\mathbf{8}$ & 'RSGA' (1) \\
\hline $\mathbf{3}$ & 'LIPL'(1) & $\mathbf{6}$ & 'LSGA'(1) & $\mathbf{9}$ & 'RIT'(1) \\
\hline
\end{tabular}

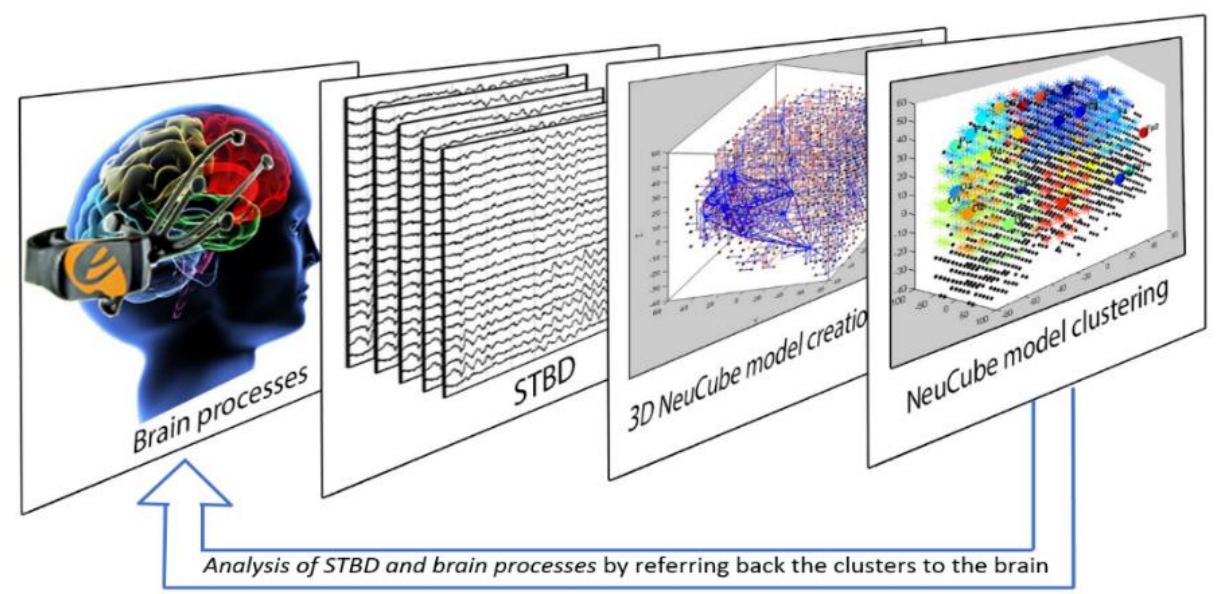

Fig. 1. The steps performed as part of the STBD clustering procedure 


\section{2 fMRI Data Mapping, Learning and Visualisation in a SNNc}

The whole fMRI set of voxels is spatially mapped into the SNNc. The spatio-temporal patterns of the 20 pre-selected voxels are encoded to sequences of spikes using TBR and later transferred to the SNNc via 20 spatially allocated input neurons. During the NeuCube training, the post synaptic potential of each neuron $n_{i}$ at time $t, \operatorname{PSPi}(\mathrm{t})$, increases by every input spike received from all pre-synaptic neurons [12]. Once the PSPi ( $\mathrm{t}$ ) exceeds a firing threshold, neuron $n_{i}$ emits a spike. Based on the STDP learning rule, if neuron $n_{i}$ spikes first and then $n_{j}$ spikes, the connection weight between these neurons increases, otherwise it decreases. After the NeuCube unsupervised learning is completed, the spatio-temporal relationships between fMRI input streams are reflected on the created neuronal connections (Fig. 2). These connections are generated differently for each of the two stimuli revealing the fact that the subject is performing differently when processing affirmative versus negative sentences. Fig. 2 shows that more and stronger neural connections are created in the left hemisphere (LDLPFC and LT) than in the right hemisphere (RDLPFC and RT) while reading negative sentences.

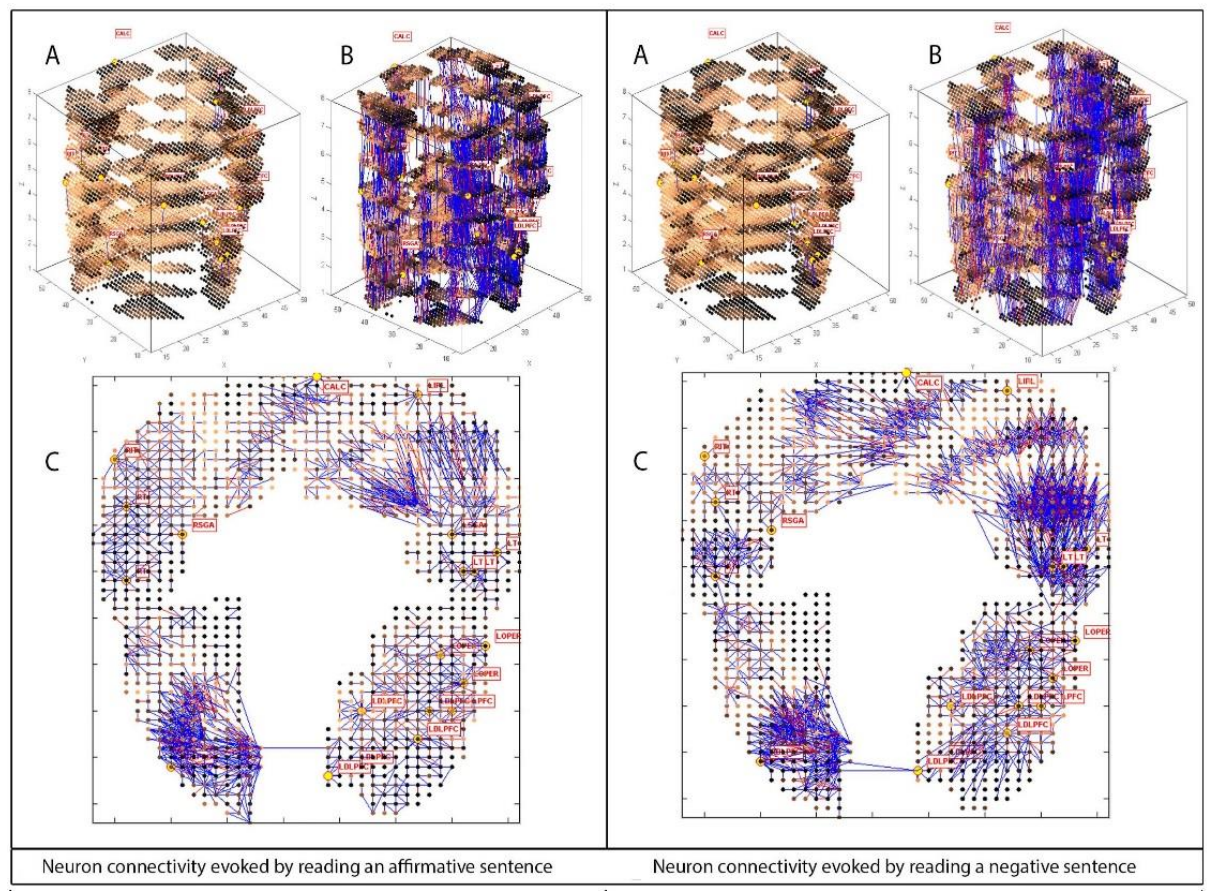

Fig. 2. Mapping of $5062 \mathrm{fMRI}$ voxels to the SNNc and the spatio-temporal connections which evolved around 20 input voxels against affirmative /negative sentence presentation. Blue lines represent positive connection weights while red lines represent negative weights. The brighter the colour of a neuron, the stronger its activity is with neighbouring neurons. Thickness of the lines also identifies the neuron's enhanced connectivity. (a) 3D visualisation of the initial connections before the NeuCube training process; (b) Connections after the NeuCube training process; (c) 2D visualisation of the spatio-temporal connections after the NeuCube training 


\subsection{Dynamic Cluster Evolution in a NeuCube Model on the fMRI Case Study STBD}

During the learning procedure, 3D neuronal clusters are evolved when new input fMRI vectors are entered and learned in the SNNc. Step-wise visualisation of the clusters evolution reveals differences of spike-time relationships between fMRI voxel activity patterns against sentence polarities. Fig. 3 shows the process of cluster creation over time for 16 selected time points during unsupervised learning of fMRI data.

(a)
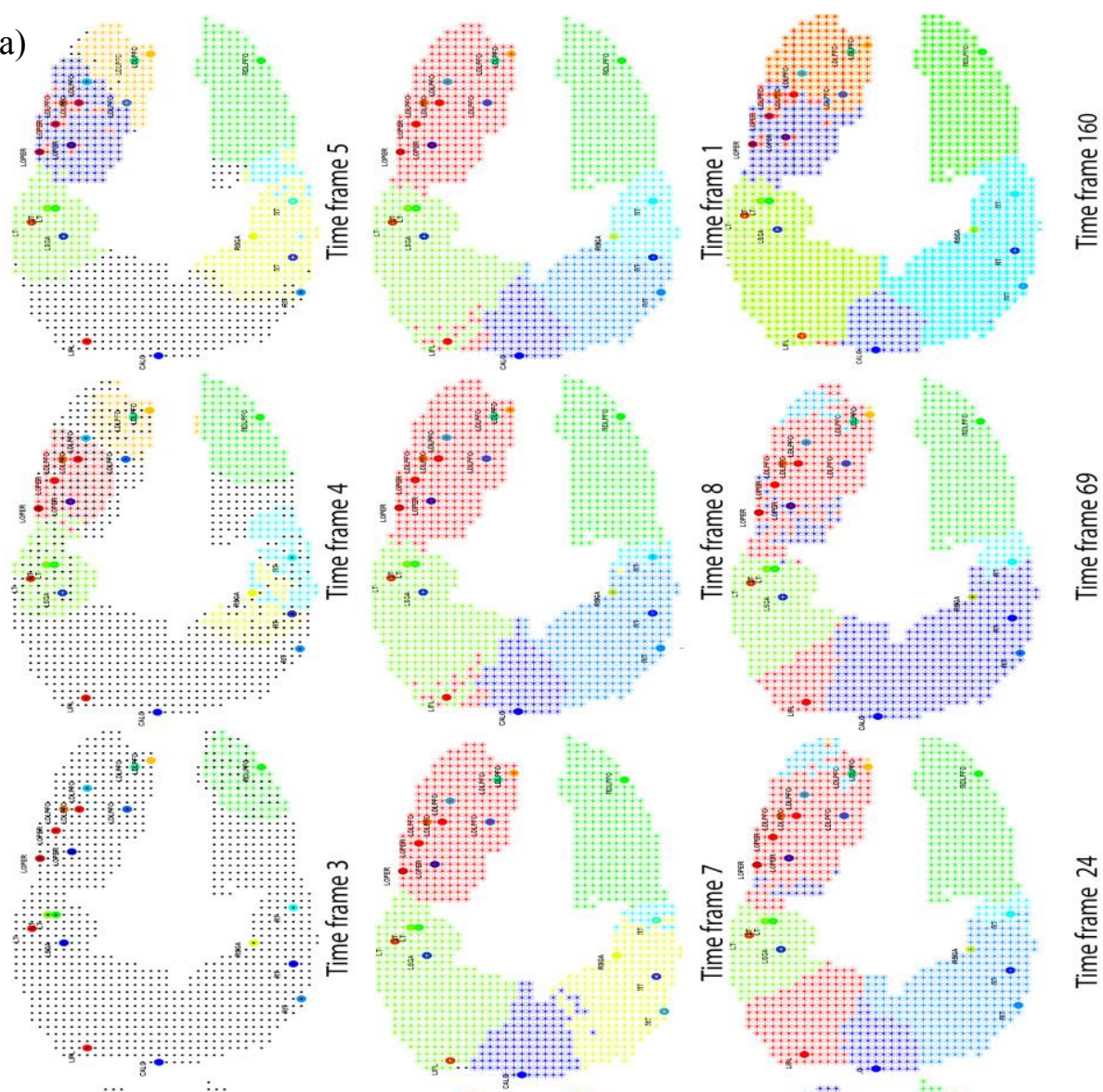

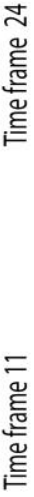
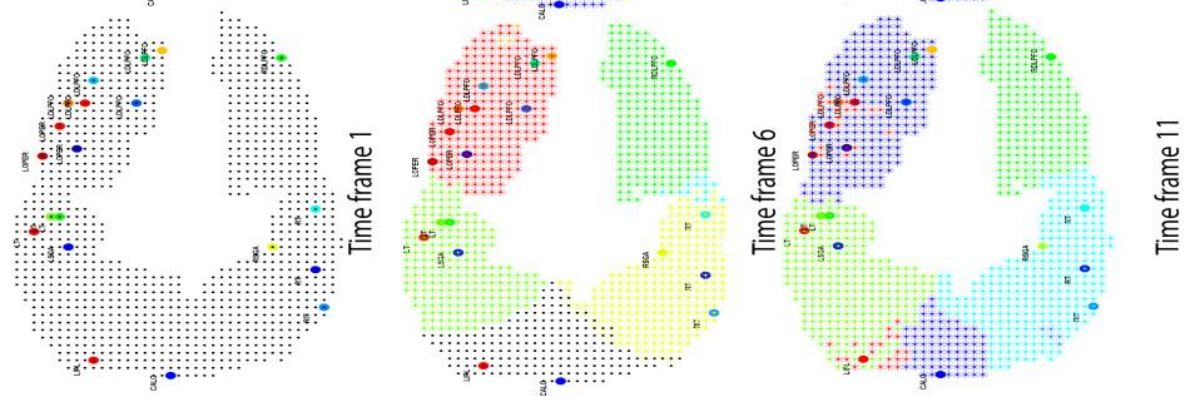

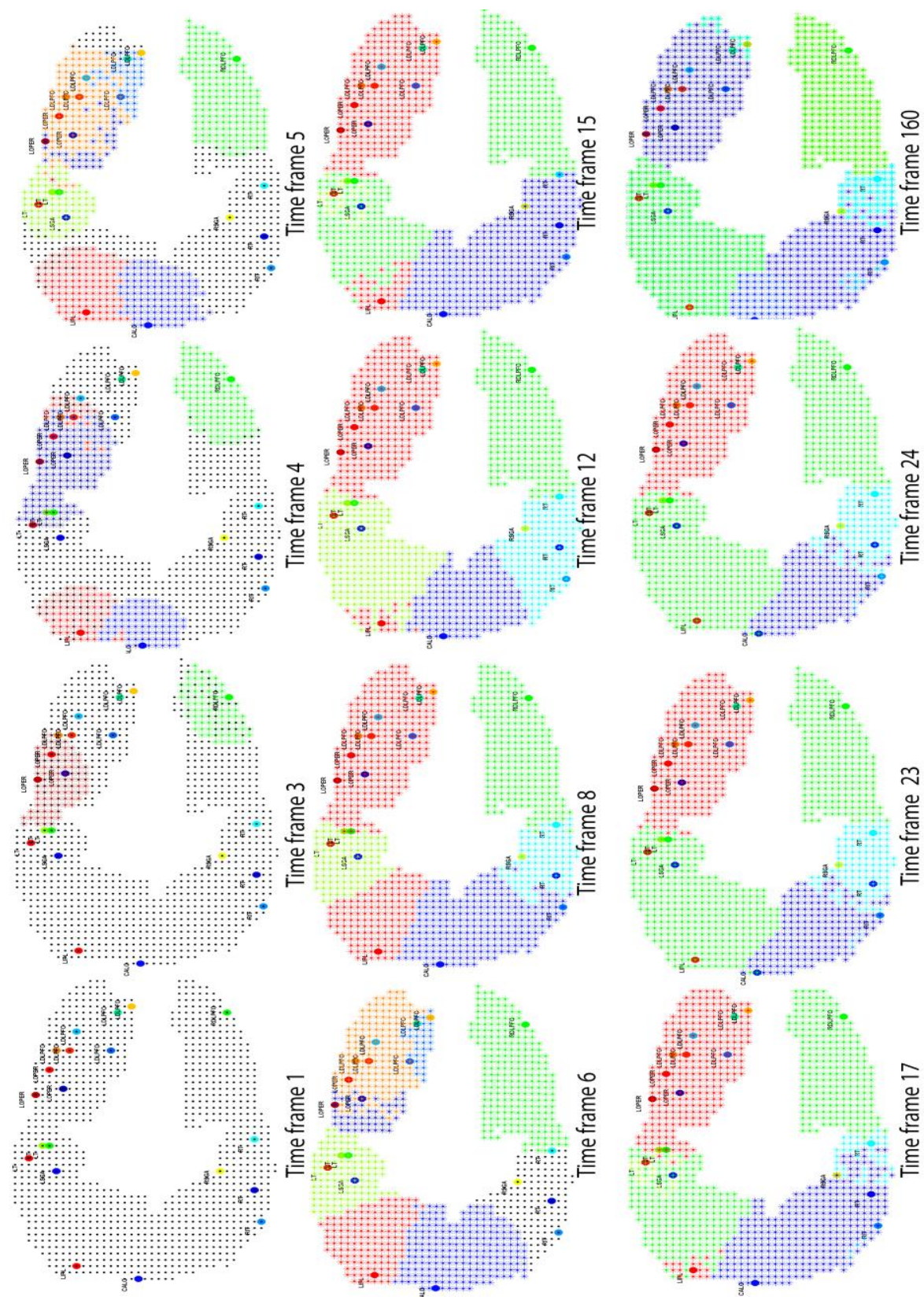

Fig. 3. (a) Step-wise visualisation of the dynamic neuronal cluster evolution corresponding to 20 voxels, while the subject was reading affirmative sentences. The first neuronal cluster is created at the $3^{\text {rd }}$ time frame of the fMRI data during the unsupervised learning in a SNNc and is associated with the RDLPFC brain region; (b) Dynamic neuronal cluster evolution while the subject was reading negative sentences. The first neuronal clusters are created at the $3^{\text {rd }}$ time frame of the learning procedure associated with the RDLPFC and LOPER brain regions 
During the NeuCube learning, there are step-wise changes in the evolution of the clusters. When a SNNc was training with fMRI data of affirmative sentences, the first produced clusters corresponded to the RDLPFC brain region after the $3^{\text {rd }}$ fMRI time frame was learned. In the case of negative sentences, the first created neuronal clusters related to RDLPFC and LOPER regions and were created after the $3^{\text {rd }}$ fMRI data frame was learned. Fig. 4 illustrates how the size of the cluster is changing in terms of the number of neurons belong to each cluster.

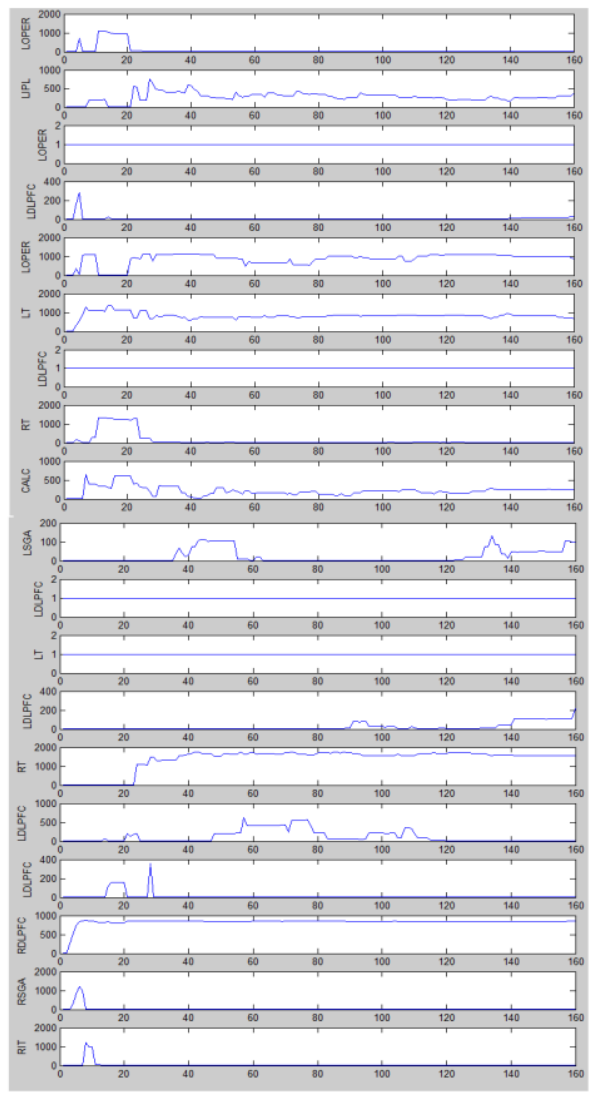

(a)

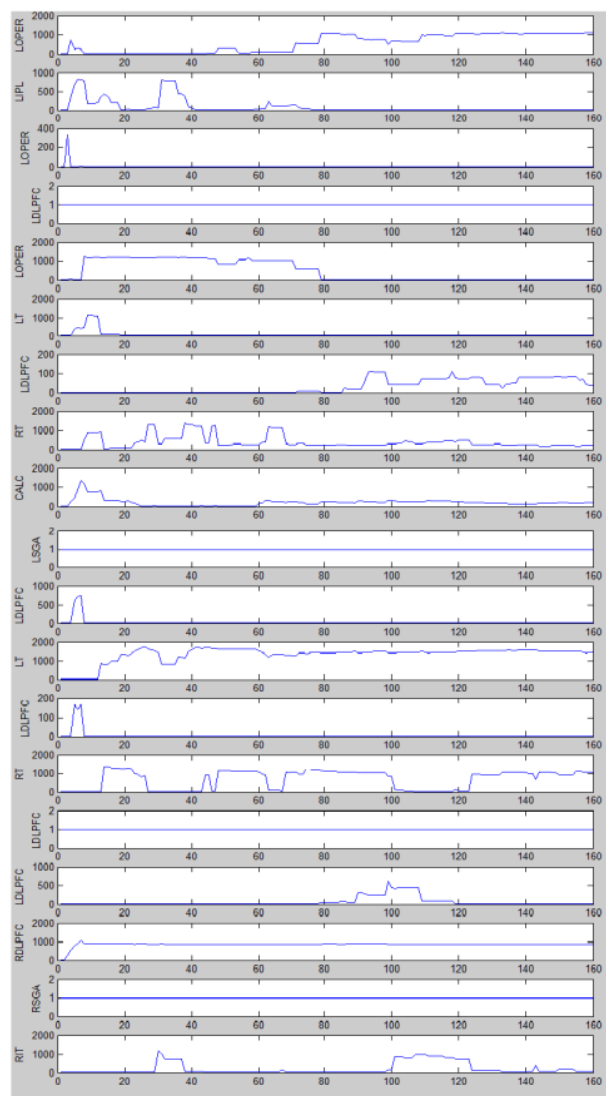

(b)

Fig. 4. Changings of the neuronal cluster size during their creation in the NeuCube SNNc while training on fMRI STBD against (a) affirmative versus (b) negative sentences

\section{Conclusion}

In this study, a generic SNN methodology was proposed as a novel method for 3D dynamic neuronal clustering of STBD. The method is based on the following scheme:

Brain processes $\rightarrow \mathrm{STBD} \rightarrow 3 \mathrm{D}$ NeuCube model creation $\rightarrow 3 \mathrm{D}$ NeuCube model clustering $\rightarrow$ Analysis of STBD $\rightarrow$ Analysis of spatio-temporal brain processes. 
The method is illustrated on a benchmark fMRI STBD. The clustering procedure uses STDP learning rule as part of unsupervised learning in a 3D SNN NeuCube -based model. The proposed method for dynamic clustering of STBD is illustrated on fMRI data, but can be applied on EEG and other STBD to study dynamic functional processes in the brain during cognitive tasks.

\section{Acknowledgment}

The research is supported by the Knowledge Engineering and Discovery Research Institute of the Auckland University of Technology (www.kedri.aut.ac.nz). The Authors would like to acknowledge the following participants and researchers that have contributed to the realisation of this study: Dr Enmei Tu, Lei Zhou, Joyce D’Mello.

\section{References}

1. Gholami-Doborjeh, M., Capecci, E., Kasabov, N.: Classification and segmentation of fMRI Spatio-Temporal Brain Data with a NeuCube Evolving Spiking Neural Network Model. IEEE SSCI, 73-80 (2014)

2. Kasabov, N., Capecci, E.: Spiking neural network methodology for modelling, classification and understanding of EEG spatio-temporal data measuring cognitive processes. Information Sciences 294, 565-575 (2014)

3. Kasabov, N.: NeuCube: A spiking neural network architecture for mapping, learning and understanding of spatio-temporal brain data. Neural Networks 52, $62-76$ (2014)

4. Indiveri, G., Linares-Barranco, B., Hamilton, T. J., Van Schaik A., Etienne-Cummings R., Delbruck, T., Liu, S.-C., Dudek, P., Hfliger, P., Renaud, S.: Neuromorphic silicon neuron circuits. Frontiers in neuroscience 5, (2011)

5. Talairach, G., Tournoux, J.P.: Co-planar stereotaxic atlas of the human brain. 3-Dimensional proportional system: an approach to cerebral imaging. Thieme Medical Publishers, (1988)

6. Brett, M., Christoff, K., Cusack, R., Lancaster, J.: Using the Talairach atlas with the MNI template. Neuroimage 13(6), 85-85 (2001)

7. Tu, E., Kasabov, N., Othman, M., Li, Y., Worner, S., Yang, J., Jia, Z.: NeuCube(ST) for Spatio-Temporal Data Predictive Modelling with a Case Study on Ecological Data. Neural Networks (IJCNN), (2014)

8. Song S., Miller, K. D., Abbott, L. F.: Competitive Hebbian learning through spike-timingdependent synaptic plasticity. Nature neuroscience 3(9), 919-926 (2000)

9. Kasabov, N., Song, Q.: DENFIS: dynamic evolving neural-fuzzy inference system and its application for time-series prediction. IEEE Transactions on Fuzzy Systems 10(2), 2, 144 154 (2002)

10. Deboeck, G., Kohonen, T.: Visual explorations in finance: with self-organizing maps. Springer London, (1998)

11. Just, M.: StarPlus fMRI data," [Online]. Available: http://www.cs.cmu.edu/afs/cs.cmu.edu/project/theo-81/www/. [Accessed 1307 2014].

12. Kasabov, N.: Neural Networks Letter: To Spike or Not to Spike: A Probabilistic Spiking Neuron Model. Neural Network 23(1), 16-19 (2010) 